> Le rôle des lymphocytes B (LB) dans l'immunosurveillance des tumeurs a longtemps été négligé car il a été souvent considéré comme peu efficace, voire pro-tumoral. Des études approfondies du microenvironnement immunitaire, notamment dans les cancers humains, ont permis de préciser la nature des interactions entre le LB et ses partenaires cellulaires. Cette revue examine un certain nombre de paramètres qui dictent le devenir du LB vers une fonction pro- ou anti-tumorale. Ainsi, la capacité à élaborer une immunité antitumorale qui repose sur les lymphocytes B, et/ ou des anticorps qu'ils sécrètent, fait appel à une palette très variée de mécanismes moléculaires et cellulaires dont certains pourraient représenter de nouvelles cibles thérapeutiques en oncologie. <

Les études sur le rôle des anticorps et des lymphocytes B (LB) dans le développement et le contrôle des tumeurs se sont développées grâce à plusieurs découvertes majeures.

Ce sont d'abord Jules Héricourt et Charles Richet qui ont mis en évidence, en 1895, le rôle anti-tumoral de sérums. En effet, l'injection de sérums de deux chiens et d'un âne immunisés contre des extraits d'ostéosarcomes à deux patients présentant une tumeur abdominale ou thoracique a montré des réponses cliniques importantes chez ces patients, marquées par une diminution significative des masses tumorales et une amélioration de leur état général [1]. Ces expériences furent suivies par la démonstration que le transfert de sérums de lapins immunisés contre la toxine diphtérique ou contre la toxine tétanique, par injection d'une dose non létale de celles-ci, permettait de protéger des animaux contre une injection létale de ces toxines [2]. Toutes ces remarquables expériences ont jeté les fondements de la sérothérapie moderne [1] et de l'utilisation des anticorps comme molécules thérapeutiques.

Ce n'est cependant qu'en 1971 que la découverte d'antigènes associés aux tumeurs (TAA, pour tumor-asso-

\section{Quel avenir pour les lymphocytes $B$ infiltrant les tumeurs solides}

\section{Marqueur pronostique et/ ou cible thérapeutique?}

Hélène Kaplon ${ }^{1-3}$, Marie-Caroline Dieu-Nosjean ${ }^{1-3}$

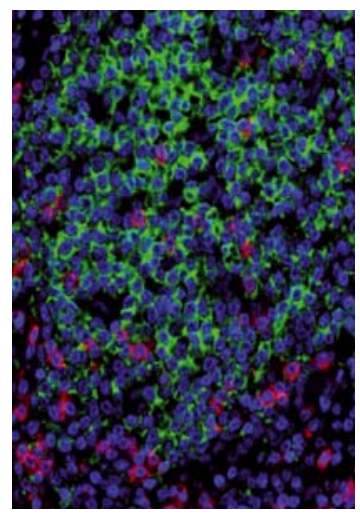

${ }^{1}$ Inserm, UMRS 1138 , Centre de recherche des cordeliers, laboratoire « Cancer et Immunité anti-tumorale », F-75006, Paris, France. ${ }^{2}$ Université Paris Descartes, Sorbonne Paris Cité, UMRS 1138, Centre de recherche des cordeliers, 15, rue de l'École de Médecine, F-75006, Paris, France. ${ }^{3}$ Université Pierre et Marie Curie (UPMC), Paris 06, Sorbonne Université, UMRS 1138 ,

ciated antigen) va stimuler un grand Centre de recherche des cordeliers, 15, rue de l'École de Médecine, F-75006, Paris, France. marie-caroline.dieu-nosjean@ inserm.fr

helene.kaplon@crc.jussieu.fr à l'élaboration de nombreux anticorps monoclonaux à usage thérapeutique dirigés contre les cellules tumorales. Un pas supplémentaire sera franchi lorsque des peptides dérivés d'antigènes spécifiques de tumeurs ou associés à des tumeurs, capables de stimuler une réponse lymphocytaire $T$, seront découverts grâce aux travaux pionniers de Thierry Boon et ses collègues [3]. De nombreux antigènes associés aux tumeurs ont désormais été identifiés par diverses approches reposant soit sur des analyses de la réponse humorale soit sur l'exploration de la réponse lymphocytaire T. L'utilisation de la technique SEREX (serological analysis of recombinant tumor cDNA expression libraries), qui repose sur le criblage de banques d'expression d'ADNc de biopsies tumorales avec le sérum des patients cancéreux correspondants, a permis notamment la découverte de molécules comme NY-ESO-1 (ou CTAGIB, cancer/testis antigen 1B) [4].

Alors que les lymphocytes $T$ ont fait l'objet d'analyses cellulaires et moléculaires détaillées, du fait de leurs fonctions effectrices, au premier rang desquelles leur capacité cytotoxique, et du fait de leur valeur pronostique favorable observée dans la majorité des cancers, les lymphocytes B sont néanmoins longtemps restés les parents pauvres des études sur l'immunité anti-tumorale. Ce n'est qu'au cours de la dernière décennie que ces cellules ont fait l'objet d'un renouveau 


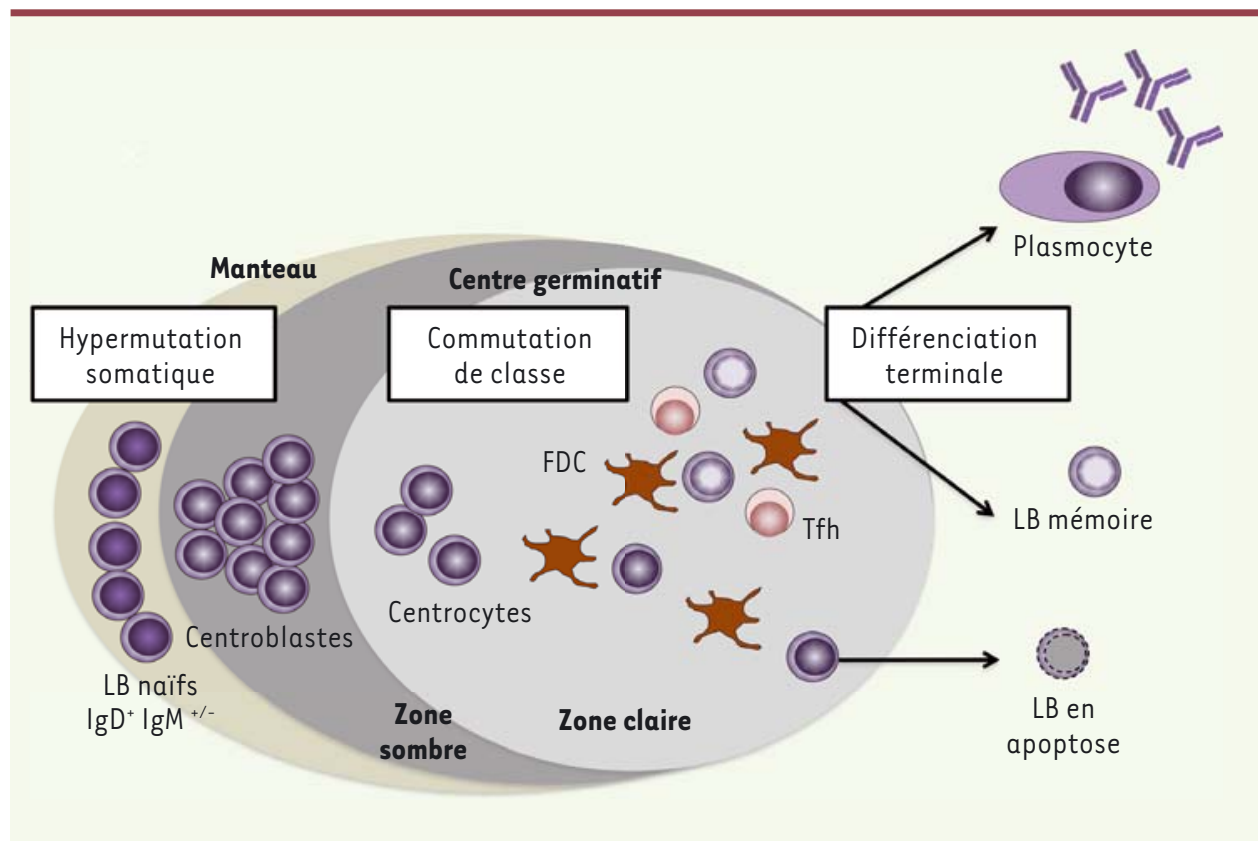

Figure 1. Processus de différenciation des lymphocytes B au sein du follicule $B$ d'un organe lymphoïde secondaire ou d'une structure lymphoïde tertiaire. Les lymphocytes B (LB) naïfs ayant rencontré l'antigène qu'ils reconnaissent vont entrer dans une phase active de prolifération, puis se différencier en centroblastes au sein de la zone sombre du centre germinatif (CG). Ces derniers vont alors subir un processus d'hypermutation somatique qui consiste en l'introduction de mutations somatiques dans les domaines variables des immunoglobulines, modifiant ainsi éventuellement l'affinité du récepteur pour l'antigène $(B C R)$.

Les centroblastes vont ensuite migrer dans la zone claire du CG pour se différencier en centrocytes. Dans cette zone, ils rencontreront des cellules dendritiques folliculaires (FDC) présentant des antigènes sous la forme de complexes immuns. Les centrocytes ayant un BCR peu affin pour l'antigène mourront par apoptose. Les autres, ainsi sélectionnés en raison de la haute affinité de leur $B C R$ muté pour l'antigène, recevront des signaux de co-stimulation délivrés par les lymphocytes T CD4 $4^{+}$folliculaires helper (Tfh) ; parallèlement débute la commutation de classe du LB. Ce processus de réarrangement génétique permet aux LB de produire une nouvelle classe d'immunoglobuline ( $\lg$, IgA, IgE) tout en conservant les mêmes domaines variables (VH et VL) et donc la spécificité de leur BCR vis-à-vis de l'antigène. Le LB achèvera alors sa différenciation en plasmocyte ou en LB mémoire.

d'intérêt significatif. Nous décrirons donc ici les données récemment obtenues sur le rôle des lymphocytes $B$ et des anticorps qu'ils sécrètent dans le développement tumoral et son contrôle, et nous discuterons notamment de leurs possibles fonctions au sein du microenvironnement tumoral, en particulier chez les patients.

\section{Ontogénie des lymphocytes B}

Après avoir effectué leurs premières étapes de maturation dans la moelle osseuse, les LB naïfs rejoignent les organes lymphoïdes secondaires où ils pourront rencontrer l'antigène dont ils sont spécifiques. Un dialogue à trois entre LB, lymphocyte T (LT) auxiliaire (ou «helper », Th) et antigène natif présenté sous forme d'un complexe immun par les cellules dendritiques folliculaires, permettra de sélectionner un LB. Ce dernier va alors avoir une phase d'expansion clonale au sein du centre germinatif (CG). Les cellules ainsi produites vont se différencier en LB mémoire ou en plasmocyte (Figure 1). Les $L B$ mémoires résideront essentiellement au niveau de la rate et des ganglions lymphatiques et seront à l'origine du développement très rapide d'une réponse humorale en cas de réexposition au même antigène. Les plasmocytes seront quant à eux localisés majoritairement dans la moelle osseuse et la lamina propria de la muqueuse intestinale.

\section{Des lymphocytes B et des anticorps qui favorisent le développement tumoral}

Les lymphocytes $B$ ont longtemps été considérés comme induisant des réponses peu efficaces vis-à-vis d'antigènes tumoraux. La présence d'anticorps spécifiques est souvent observée dans le sérum des patients cancéreux. Cependant, la plupart de ces anticorps sont dirigés contre des protéines cytoplasmiques ubiquitaires libérées au cours de la mort des cellules tumorales. Ainsi, ces anticorps sont peu susceptibles d'exercer une activité anti-tumorale directe, du fait de leur incapacité à pénétrer les cellules tumorales vivantes et à se fixer à leur cible intracellulaire [5].

De plus, l'utilisation de souris transgéniques exprimant des gènes du papillomavirus humain de type 16 sous le contrôle du promoteur kératine 14 (K14-HPV16), a mis en évidence que, dans ce modèle, les anticorps étaient les médiateurs par lesquels les lymphocytes $B$ peuvent favoriser la progression tumorale. En effet, la présence d'immuns complexes dans le tissu pré-cancéreux a induit: (1) une réponse inflammatoire aiguë caractérisée par une augmentation significative du recrutement 
de cellules immunitaires; (2) l'activation par les complexes immuns des récepteurs pour la région $\mathrm{Fc}$ des IgG (RFc $\gamma$, ou Fc $\gamma R$ en anglais) des mastocytes et des macrophages résidants et recrutés modulent leur fonction pro-angiogénique et protumorigène, ce qui contribue à la carcinogenèse squameuse [6].

Par ailleurs, les IgG4 - l'une des sous-classes d'IgG chez l'homme -, produites contre les tumeurs, pourraient empêcher la fixation d'autres sous-classes présentant des propriétés effectrices puissantes comme les IgGl, avec pour conséquence de limiter la cytotoxicité cellulaire dépendante des anticorps (ADCC, antibody-dependent cellular cytotoxicity) [7] et, potentiellement, l'activation de la voie classique du complément. Ainsi, différentes observations suggèrent que, selon le contexte tumoral, les anticorps produits lors du développement des tumeurs pourraient avoir un rôle pro-tumoral, direct ou indirect.

Mais qu'en est-il des LB eux-mêmes ? Différents modèles murins ont clairement mis en évidence un rôle pro-tumoral de ces cellules [8, 9]. En effet, la déplétion des LB chez des souris sauvages conduit à une augmentation de la production d'IFN- $\gamma$ (interféron $\gamma$ ) par les LT $\mathrm{CD}^{+}$et les cellules NK (natural killer) comparativement à des souris contrôles. La présence de LB dits régulateurs (Breg) producteurs d'IL(interleukine)-10 a effectivement été rapportée par Mizoguchi et al. [10]. Les Breg sont capables d'inhiber la prolifération des LT CD4 ${ }^{+}$ en sécrétant de I'IL-10 et d'inhiber la production de cytokines proinflammatoires (IL-1 $\beta$ et TNF- $\alpha$ [tumor necrosis factor- $\alpha$ ], IL-12, IFN- $\gamma$ ) par les macrophages, les cellules dendritiques et les cellules NK. Par ailleurs, les LB peuvent induire l'apoptose des LT CD4 $4^{+}$en exprimant le ligand de Fas (FasL) qui va alors se lier à la molécule Fas exprimée à la surface de ces LT, ou encore provoquer l'anergie des LT CD $8^{+}$[11]. Shalapour et al. ont ainsi montré dans un modèle murin de cancer de la prostate résistant à la castration que le traitement par l'oxaliplatine induisait un recrutement de plasmocytes IgA+ exprimant PD-LI (programmed death-ligand 1 ) et sécrétant de I'IL-10 dans les tumeurs, entraînant l'inhibition des fonctions cytotoxiques des LT CD8 ${ }^{+}$PD $-1^{+}$ (programmed cell death 1) [12]. Dans ces conditions expérimentales, les plasmocytes contribuent activement à l'élaboration d'un environnement pro-tumoral. D'autres cytokines produites par les $L B$, telles que I'IL-35 et le TGF- $\beta$ (transforming growth factor-beta), ont également été décrites pour leur effet immunosuppressif. Enfin, la qualité de l'interaction LB-LT est également déterminante sur la fonctionnalité du LT. Une très faible expression de molécules de co-stimulation par les LB intra-tumoraux (LB dits non activés) a été rapportée dans plusieurs modèles: cette faible expression conduit à l'anergie des LT infiltrant les tumeurs $[13,14]$.

\section{Les lymphocytes B comme acteurs de l'immunité anti- tumorale}

Le rôle pro-tumoral des lymphocytes B est cependant controversé. II s'appuie essentiellement sur des études menées chez la souris, utilisant des modèles tumoraux dont la pertinence avec les situations tumorales rencontrées chez les patients est discutable. En fait, des analyses récentes de l'infiltrat $B$ menées chez des patients présentant différents types de tumeurs ont démontré que la présence d'une forte densité de LB est associée à un meilleur pronostic dans de nombreux cancers solides [15, 16]. Les LB semblent donc jouer un rôle anti-tumoral significatif bien moins marginal que celui initialement pensé dans l'élaboration de réponses immunitaires anti-tumorales chez les patients. Leur faible nombre dans le microenvironnement tumoral de la plupart des tumeurs solides, comparativement à ceux des lymphocytes $T$ et des cellules myéloïdes, suggère des modes d'action puissants et multiples.

Parmi les différents mécanismes pouvant rendre compte de ce rôle anti-tumoral, le rôle de LB comme cellules présentatrices d'antigènes (CPA) a été analysé en détail [14]. En effet, dans les cancers du sein et de l'ovaire, les LB intra-tumoraux expriment à leur surface des niveaux élevés de molécules de co-stimulation (comme par exemple CD40, CD80, CD86) et du complexe majeur d'histocompatibilité de classe II (CMH-II) caractéristiques des CPA [17]. Dans le cancer ovarien de haut grade, Nielsen et al. ont montré que la coopération entre les LB et les $L T$ joue un rôle essentiel dans l'induction d'une réponse anti-tumorale, entraînant un bénéfice en terme de survie [17]. De façon très intéressante, ces mêmes auteurs ont démontré que l'infiltrat intra-tumoral de plasmocytes était positivement corrélé avec ceux des $\mathrm{LT} C D 8^{+}$et des $\mathrm{LB}$, ces derniers étant exclusivement retrouvés au sein des structures lymphoïdes tertiaires (TLS) associées à la tumeur [18]. De

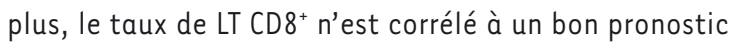
qu'en présence d'une forte densité de plasmocytes intra-tumoraux, suggérant une coopération étroite entre ces deux populations immunitaires. Une collaboration LB et $L T$ conduisant à une réponse anti-tumorale significative a également été mise en évidence dans le carcinome hépatocellulaire [19]. Tous ces résultats sont en accord avec d'autres travaux menés chez la souris démontrant que la déplétion des $L B$ conduit à un mauvais «priming » des $\mathrm{LT} C D 4^{+}$et $\mathrm{CD} 8^{+}$et provoque une diminution de l'activation des LT intra-tumoraux [19]. Ce dialogue entre LB et LT va également influencer le profil de sécrétion des cytokines par les LB eux-mêmes que I'on peut alors classer en trois sous-groupes: les Breg sécrétant des cytokines immunosuppressives, les lymphocytes Be2 ( $B$ effector-2) sécrétant des cytokines (IL2, IL-4, TNF- $\alpha$, IL-6) impliquées dans les réponses Th2, et les lymphocytes Bel sécrétant des cytokines (IFN- $\gamma$, IL-12, TNF- $\alpha$ ) impliquées dans la polarisation Thl [20]. Shi et al. ont montré que les cellules Bel sont prépondérantes dans le carcinome hépatocellulaire et ont suggéré que ces cellules pourraient participer activement au développement d'une réponse anti-tumorale Thl [16]. 


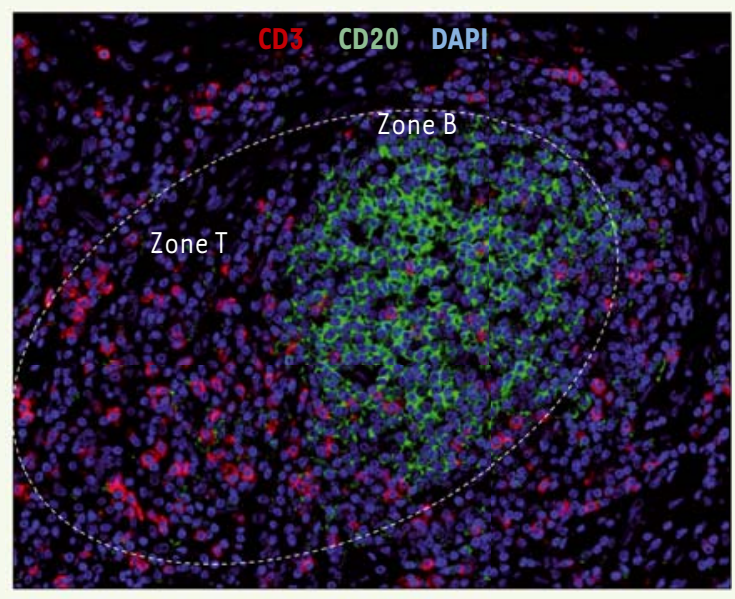

Figure 2. Ségrégation lymphocytaire au sein d'une structure lymphoïde tertiaire. Exemple d'un TLS (structure lymphoïde tertiaire) dans le cancer du poumon non à petites cellules. Triple marquage en immunofluorescence sur une coupe de tissu fixé et inclus en paraffine. Les TLS, localisées dans le stroma tumoral, présentent une zone $\mathrm{B}$ riche en lymphocytes $\mathrm{B} C D 20^{+}$(en vert) adjacente à une zone $T$ riche en lymphocytes $T \mathrm{CD}^{+}$(en rouge). Cette organisation cellulaire est similaire à celle observée dans les organes lymphoïdes secondaires (grossissement $x$ 200).

Différents modèles murins ont par ailleurs montré que des LB activés sont capables d'induire de façon directe et indirecte la prolifération des LT CD8 ${ }^{+}$et de contrôler ainsi la croissance tumorale [21]. À l'instar des certaines cellules dendritiques, certains LB seraient capables de réaliser une présentation dite «croisée », en présentant des peptides antigéniques à des LT CD8 ${ }^{+}$dans un contexte de CMH de classe I [22, 23]. Un second mécanisme a été décrit où l'engagement de l'axe de co-stimulation CD27/CD70 peut conduire à la prolifération et à la survie des $\mathrm{LT} C D 8^{+}$indépendamment de l'antigène. Ces résultats mettent ainsi en évidence le rôle auxiliaire (ou helper) important que jouent les LB dans la mise en place d'une réponse T cytotoxique [24]. Cependant, l'existence d'un tel mécanisme n'a pas été démontrée à ce jour au sein du microenvironnement tumoral.

Les plasmocytes représentent une composante importante de la réponse anti-tumorale. Leur différenciation peut s'effectuer au niveau d'un organe lymphoïde secondaire et/ou au sein des TLS associées aux tumeurs $[15,18]$. La valeur pronostique des plasmocytes reste sujette à controverse dans les cancers solides (Tableau I). Ceci pourrait être dû en partie à la diversité des marqueurs utilisés pour les détecter, marqueurs qui ne se recouvrent que partiellement selon les études, au manque de spécificité de certains de ces marqueurs dans certains cancers (par exemple, CD138 [syndecan-1] dans le cancer du poumon) et/ou aux différences des rôles joués par les plasmocytes en fonction du type de tumeur. À l'heure actuelle, aucun consensus n'existe quant au choix du(des) marqueur(s) à utiliser pour définir les plasmocytes (CD138, IGKC [immunoglobulin kappa chain], IgA, IgG, CD27 $7^{+} / \mathrm{CD} 38^{++}$, etc.). Dans le cancer du poumon, Lohr et al. ont montré que la production d'IGKC constitue un marqueur pronostique à l'échelle transcriptomique et protéique comparable à celle du métagène ${ }^{1}$ des LB [25]. Ainsi, une forte densité de plasmocytes $\lg \kappa \mathrm{C}^{+}$ou une forte expression d'IgKC est associée à un meilleur pronostic dans la majorité des cancers étudiés (Tableau I). Par ailleurs, l'expression d'IgKC permet de prédire la réponse aux anthracyclines chez les patientes atteintes d'un cancer

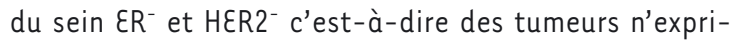
mant ni le récepteur des oestrogènes (estrogen receptor) ni le récepteur HER2/neu [26].

Plusieurs études, dont les nôtres, ont mis en évidence que des antigènes tumoraux étaient la cible des anticorps sécrétés par les plasmocytes infiltrant les tumeurs humaines $[15,36]$. Cependant, il n'a pas été démontré à ce jour que ces anticorps spécifiques jouaient un rôle anti-tumoral. La présence d'anticorps aux sites tumoraux semble néanmoins être corrélée à des réponses anti-tumorales bénéfiques. C'est ainsi qu'il a été montré dans un modèle de souris SCID (severe combined immunodeficiency) xéno-transplantées que les souris présentant une régression tumorale avaient une quantité d'IgG produites par les LB intra-tumoraux supérieure à celle des souris montrant une progression tumorale [37]. Par ailleurs, la présence de dépôts d'anticorps sur les cellules tumorales pourrait permettre à des cellules effectrices (comme les macrophages, les cellules dendritiques, les cellules NK, les neutrophiles) présentes localement d'induire de I'ADCC, de la cytotoxicité dépendante du complément ( $C D C$ ) ou encore de favoriser la phagocytose. Carmi et al. ont notamment montré que le rejet de la tumeur est dépendant d'une reconnaissance antigène-anticorps et de l'engagement des RFc $\gamma$ exprimés par les cellules dendritiques [38]. Les complexes immuns ainsi internalisés par les cellules dendritiques conduisent à l'activation de $\mathrm{LT} C D 4^{+}$et CD8 ${ }^{+}$spécifiques [39].

Un mécanisme amont par lequel les LB sont susceptibles de contrôler le développement tumoral serait leur implication dans la néo-genèse des TLS, qui sont des structures d'architecture similaire à celle des ganglions lymphatiques, composées d'une zone $B$ adjacente à une zone $T$ (Figure 2). Dans un modèle infectieux et de colite induite par le sodium dextran sulfate (DSS), l'expression de la lymphotoxine par les LB conduit à la formation des TLS chez des souris déficientes pour le facteur de transcription ROR

${ }^{1}$ Le métagène $B$ est défini comme un ensemble de gènes correspondant à la signature génique des LB et des plasmocytes. 


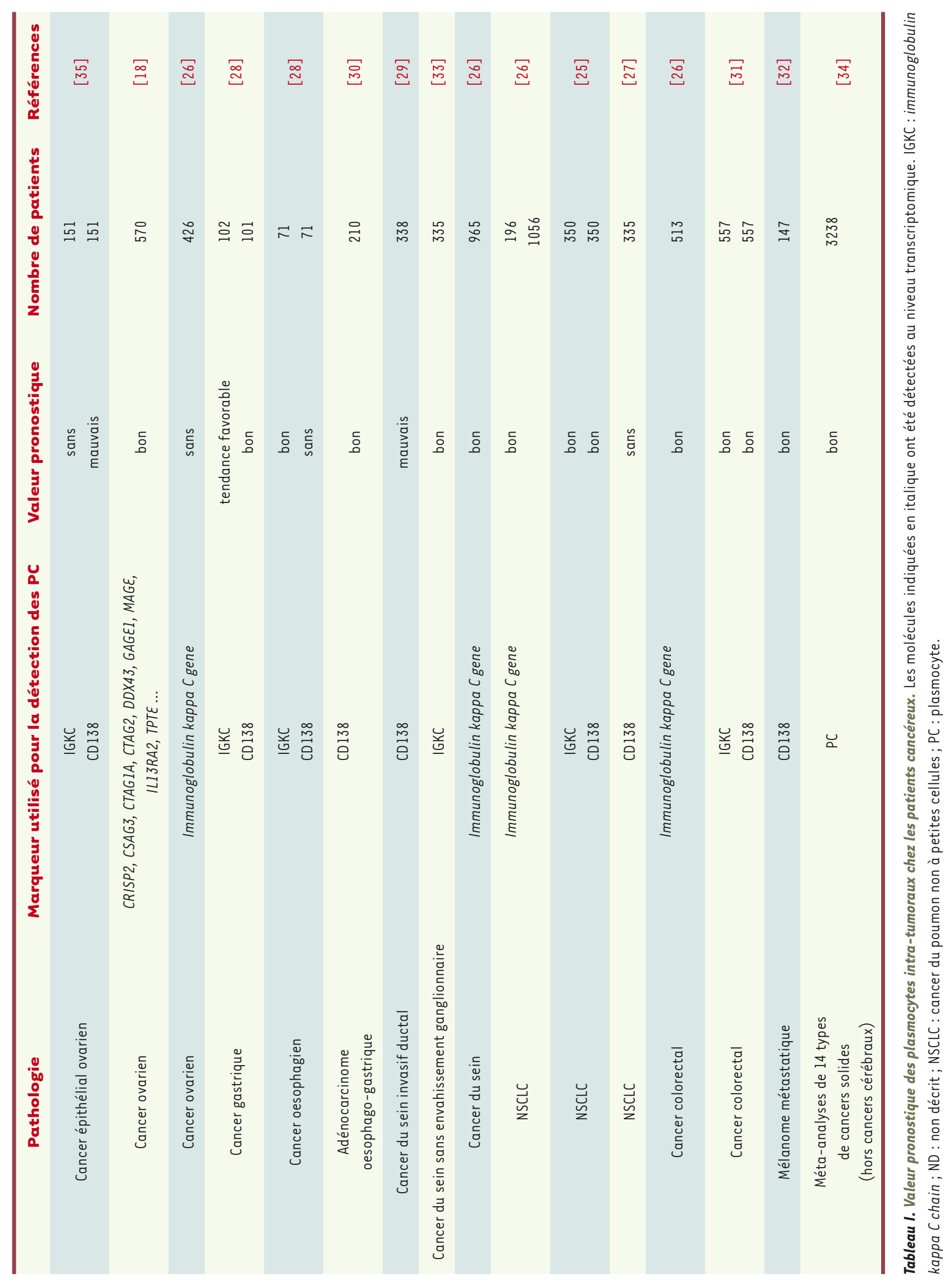




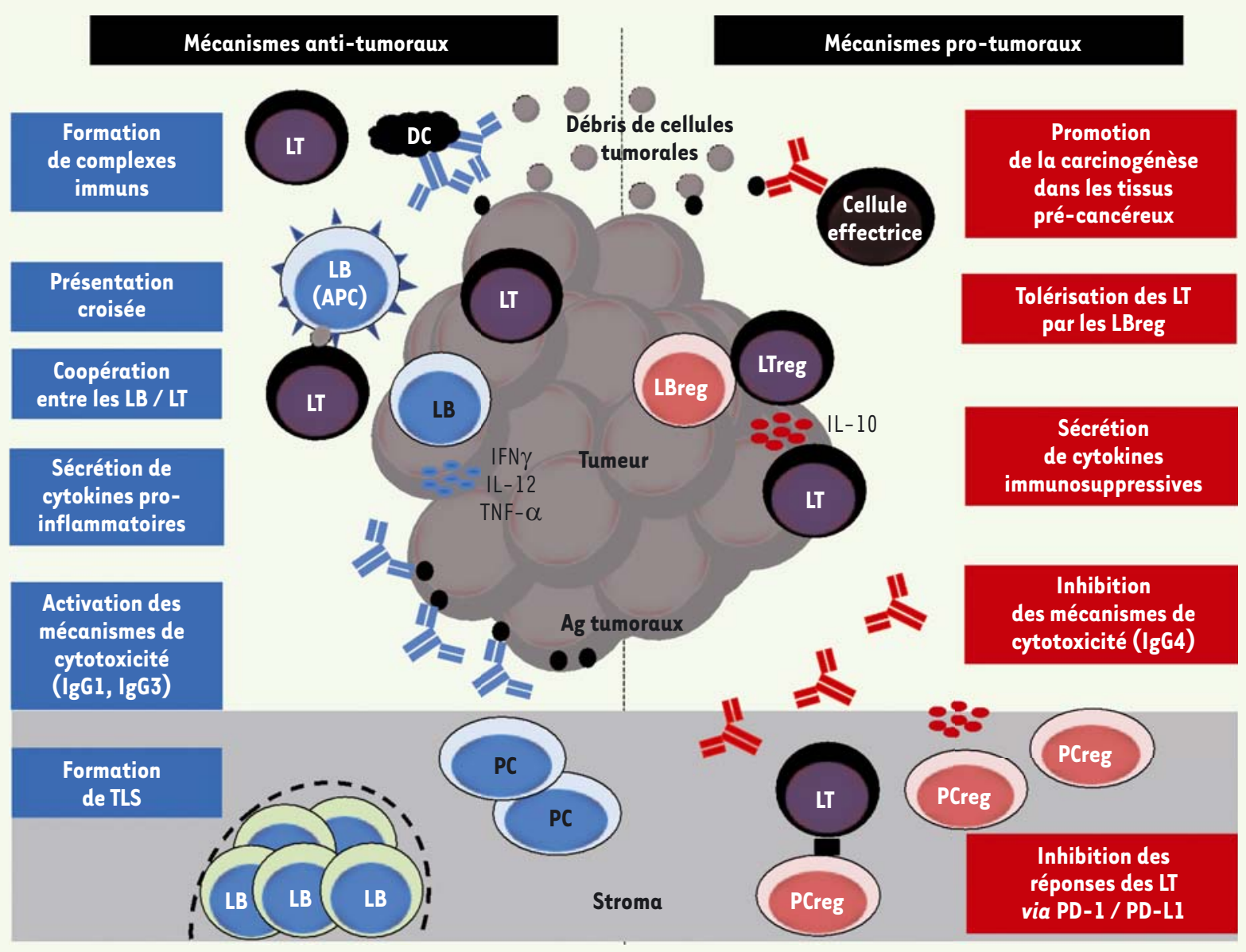

Figure 3. Mécanismes pro- versus anti-tumoraux développés par les LB infiltrant les tumeurs solides. Ag : antigène ; $A P C$ : cellule présentatrice d'antigènes ; Breg : lymphocyte B régulateur ; DC : cellule dendritique ; LB : lymphocyte B ; LT : lymphocyte T; LTreg : lymphocyte T régulateur ; PC : plasmocyte; PCreg : plasmocyte régulateur ; PD-1 : programmed cell death-1 ; PD-Ll : programmed death-ligand 1.

gamma (ROR $\gamma \mathrm{t}$ ) et en cellules initiatrices de tissus lymphoïdes $[40$, 41]. En jouant ainsi un rôle de cellules initiatrices des TLS, les LB pourraient promouvoir leur propre recrutement au sein de ces structures lymphoïdes ectopiques, et, par voie de conséquence, amplifier le développement de réactions immunitaires anti-tumorales au site même de la tumeur [15] comme le suggère très fortement la valeur pronostique favorable des LB organisés en TLS dans les cancers du poumon [15], colorectaux [42,43] et de la cavité orale [44].

\section{Conclusion}

II existe ainsi un faisceau de résultats démontrant l'importance de l'immunité humorale dans le contrôle du développement tumoral (Figure 3), comme le montre le récent article publié par Shalapour et al. [45], malgré un rôle des LB resté longtemps minoré, voire ignoré. Ces lymphocytes sont en fait des biomarqueurs favorables dans de nombreux cancers solides. Les mécanismes effecteurs mis en jeux sont de mieux en mieux caractérisés, en partie grâce à l'analyse approfon- die du microenvironnement tumoral et des interactions cellulaires qui y prennent place.

Ces données nouvelles sur le rôle des LB dans les tumeurs solides permettent désormais de se poser la question de l'impact des stratégies actuellement développées visant les points de contrôles immunitaires sur la fonctionnalité des cellules $B$ et sur la qualité de la réponse humorale vis-à-vis de la tumeur. Par exemple, PD-LI étant exprimé par les LB et les plasmocytes, et PDl par les lymphocytes Tfh (lymphocytes T folliculaires helpers), la question des conséquences de l'inhibition des interactions PDI-PD-LI par des anticorps ciblant l'une ou l'autre de ces molécules sur les activités anti- ou pro-tumorales des LB se pose. Une meilleure coordination entre immunité à médiation cellulaire et immunité humorale représente certainement un nouvel enjeu essentiel pour développer des réponses protectrices encore plus efficaces contre les tumeurs. $\diamond$ 


\section{SUMMARY}

Which future for B lymphocytes infiltrating solid tumors: prognostic biomarker and/or therapeutic target?

The role of B lymphocytes in tumor immuno-surveillance has been neglected for a long time because it has been often considered to be ineffective if not pro-tumoral. Extensive studies of the tumor immune microenvironment, particularly in humans, has now made it possible to specify the nature of the interactions between $B$ cells and their cellular partners. This review will present a number of parameters that dictate the fate of $B$ cells toward a pro-tumor versus an anti-tumor function. Thus, the ability to elicit a B cell-and/or an antibody-dependent anti-tumor immunity involves a wide variety of molecular and cellular mechanisms, some of which may represent novel therapeutic targets in oncology. $\diamond$

\section{REMERCIEMENTS}

Les auteurs remercient tous les membres du laboratoire «Cancer et immunité anti-tumorale », UMRS 1138 Inserm, Centre de Recherche des Cordeliers. Le Dr H. Kaplon a bénéficié d'une allocation doctorale de la Ligue Nationale Contre le Cancer.

\section{LIENS D’INTÉRÊT}

Les auteurs déclarent participer ou avoir participé à des interventions ponctuelles (travaux scientifiques, activités de conseil, conférences, colloques, actions de formation) pour les entreprises Astra Zeneca, BMS, Janssen, Medlmmune, MSD, Merck, Transgene.

\section{RéFÉRENCES}

1. Lahaie YM, Watier H. Contribution of physiologists to the identification of the humoral component of immunity in the $19^{\text {th }}$ century. MAbs $2017 ; 9: 774-80$.

2. Von Behring $\varepsilon$, Kitasato $S$. The mechanism of diphtheria immunity and tetanus immunity in animals (1890). Mol Immunol 1991 ; $1317: 1319-20$.

3. van der Bruggen P, Traversari C, Chomez P, et al. A gene encoding an antigen recognized by cytolytic T lymphocytes on a human melanoma. Science $1991 ; 254$ : 1643-7.

4. Chen YT, Scanlan MJ, Sahin U, et al. A testicular antigen aberrantly expressed in human cancers detected by autologous antibody screening. Proc Natl Acad Sci USA 1997 ; 94 : 1914-8.

5. Lee YT, Sheikh KM, Quismorio FP Jr, Friou GJ. Circulating anti-tumor and autoantibodies in breast carcinoma: relationship to stage and prognosis. Breast Cancer Res Treat $1985 ; 6: 57-65$.

6. Andreu P, Johansson M, Affara NI, et al. FcRgamma activation regulates inflammation-associated squamous carcinogenesis. Cancer Cell $2010 ; 17: 121-34$.

7. Karagiannis $P$, Gilbert $A \varepsilon$, Josephs $D H$, et al. IgG4 subclass antibodies impair antitumor immunity in melanoma. J Clin Invest 2013 ; 123 : 1457-74.

8. Qin Z, Richter G, Schuler T, et al. B cells inhibit induction of T cell-dependent tumor immunity. Nat Med $1998 ; 4: 627-30$.

9. de Visser KE, Korets LV, Coussens LM. De novo carcinogenesis promoted by chronic inflammation is B lymphocyte dependent. Cancer Cell $2005 ; 7: 411-23$.

10. Mizoguchi $\varepsilon$, Mizoguchi A, Preffer Fl, Bhan AK. Regulatory role of mature B cells in a murine model of inflammatory bowel disease. Int Immunol $2000 ; 12: 597-605$.

11. Rosser $\varepsilon C$, Mauri C. Regulatory B cells: origin, phenotype, and function. Immunity 2015; 42 : 607-12.

12. Shalapour S, Font-Burgada J, Di Caro G, et al. Immunosuppressive plasma cells impede T-celldependent immunogenic chemotherapy. Nature $2015 ; 521: 94-8$.

13. Watt $V$, Ronchese F, Ritchie D. Resting B cells suppress tumor immunity via an MHC class-II dependent mechanism. J Immunother $2007 ; 30: 323-32$.

14. Rodriguez-Pinto D. B cells as antigen presenting cells. Cell Immunol $2005 ; 238: 67-75$.

15. Germain C, Gnjatic S, Tamzalit F, et al. Presence of B cells in tertiary lymphoid structures is associated with a protective immunity in patients with lung cancer. Am J Respir Crit Care Med 2014 ; 189 : 832-44.

16. Shi Jy, Gao $Q$, Wang ZC, et al. Margin-infiltrating CD20+ $B$ cells display an atypical memory phenotype and correlate with favorable prognosis in hepatocellular carcinoma. Clin Cancer Res 2013 ; $19: 5994-6005$.

17. Nielsen JS, Nelson BH. Tumor-infiltrating B cells and T cells: Working together to promote patient survival. Oncoimmunology $2012 ; 1: 1623-5$.

18. Kroeger DR, Milne K, Nelson BH. Tumor-infiltrating plasma cells are associated with tertiary lymphoid structures, cytolytic $\mathrm{t}$-cell responses, and superior prognosis in ovarian cancer. Clin Cancer Res $2016 ; 22: 3005-15$.

19. Garnelo M, Tan A, Her Z, et al. Interaction between tumour-infiltrating B cells and T cells controls the progression of hepatocellular carcinoma. Gut $2017 ; 66: 342-51$.

20. Lund FE. Cytokine-producing B lymphocytes-key regulators of immunity. Curr Opin Immunol 2008 ; $20: 332-8$.
21. Ritchie DS, Yang J, Hermans IF, Ronchese F. B-lymphocytes activated by CD40 ligand induce an antigen-specific anti-tumour immune response by direct and indirect activation of CD8 ${ }^{+}$T-cells. Scand J Immunol $2004 ; 60$ : 543-51.

22. Ahmadi T, Flies A, عfebera $Y$, Sherr DH. CD40 Ligand-activated, antigenspecific $B$ cells are comparable to mature dendritic cells in presenting protein antigens and major histocompatibility complex class I- and class II-binding peptides. Immunology 2008 ; $124: 129-40$.

23. Atanackovic $D$, Matsuo $M$, Ritter $\varepsilon$, et al. Monitoring $C D 4^{+} T$ cell responses against viral and tumor antigens using T cells as novel target APC. J Immunol Methods $2003 ; 278: 57-66$

24. Deola S, Panelli MC, Maric D, et al. Helper B cells promote cytotoxic T cell survival and proliferation independently of antigen presentation through CD27/CD70 interactions. J Immunol 2008 ; 180 : 1362-72.

25. Lohr M, Edlund K, Botling J, et al. The prognostic relevance of tumourinfiltrating plasma cells and immunoglobulin kappa $\mathrm{C}$ indicates an important role of the humoral immune response in non-small cell lung cancer. Cancer Lett $2013 ; 333: 222-8$.

26. Schmidt M, Hellwig B, Hammad $S$, et al. A comprehensive analysis of human gene expression profiles identifies stromal immunoglobulin kappa $C$ as a compatible prognostic marker in human solid tumors. Clin Cancer Res 2012 ; $18: 2695-703$.

27. Al-Shibli K, Al-Saad S, Andersen S, et al. The prognostic value of intraepithelial and stromal CD3-, CD117- and CD138-positive cells in nonsmall cell lung carcinoma. APMIS $2010 ; 118: 371-82$.

28. Fristedt R, Borg D, Hedner C, et al. Prognostic impact of tumour-associated $B$ cells and plasma cells in oesophageal and gastric adenocarcinoma. J Gastrointest Oncol $2016 ; 7: 848-59$.

29. Mohammed ZM, Going JJ, Edwards J, et al. The relationship between lymphocyte subsets and clinico-pathological determinants of survival in patients with primary operable invasive ductal breast cancer. Br J Cancer $2013 ; 109: 1676-84$.

30. Knief J, Reddemann K, Petrova $\varepsilon$, et al. High density of tumor-infiltrating B-lymphocytes and plasma cells signifies prolonged overall survival in adenocarcinoma of the esophagogastric junction. Anticancer Res 2016 ; 36 : 5339-45.

31. Berntsson J, Nodin B, Eberhard J, et al. Prognostic impact of tumour-infiltrating B cells and plasma cells in colorectal cancer. Int J Cancer 2016 ; 139 : 1129-39.

32. Erdag G, Schaefer JT, Smolkin ME, et al. Immunotype and immunohistologic characteristics of tumor-infiltrating immune cells are associated with clinical outcome in metastatic melanoma. Cancer Res $2012 ; 72: 1070-80$.

33. Chen Z, Gerhold-Ay A, Gebhard S, et al. Immunoglobulin kappa C predicts overall survival in node-negative breast cancer. PLoS One $2012 ; 7$ : e44741.

34. Gentles AJ, Newman AM, Liu CL, et al. The prognostic landscape of genes and infiltrating immune cells across human cancers. Nat Med $2015 ; 21$ : 938-45.

35. Lundgren S, Berntsson J, Nodin B, et al. Prognostic impact of tumour-associated B cells and plasma cells in epithelial ovarian cancer. J Ovarian Res $2016 ; 9: 21$.

36. Pavoni $\varepsilon$, Monteriu G, Santapaola D, et al. Tumor-infiltrating B lymphocytes as an efficient source of highly specific immunoglobulins recognizing tumor cells. BMC Biotechnol $2007 ; 7: 70$.

37. Mizukami M, Hanagiri T, Shigematsu $Y$, et al. Effect of IgG produced by tumorinfiltrating B lymphocytes on lung tumor growth. Anticancer Res $2006 ; 26$ : 1827-31.

38. Carmi Y, Spitzer MH, Linde IL, et al. Allogeneic IgG combined with dendritic cell stimuli induce antitumour T-cell immunity. Nature 2015; 521 : 99-104.

39. Rafiq K, Bergtold A, Clynes R. Immune complex-mediated antigen presentation induces tumor immunity. J Clin Invest $2002 ; 110$ : 71-9.

40. Lochner M, Ohnmacht C, Presley L, et al. Microbiota-induced tertiary lymphoid tissues aggravate inflammatory disease in the absence of RORgamma t and LTi cells. J Exp Med 2011 ; 208 : 125-34.

41. McDonald KG, McDonough JS, Newberry RD. Adaptive immune responses are dispensable for isolated lymphoid follicle formation: antigen-naive, lymphotoxin-sufficient $B$ lymphocytes drive the formation of mature isolated lymphoid follicles. J Immunol $2005 ; 174: 5720-8$.

42. Bindea $G$, Mlecnik $B$, Tosolini $M$, et al. Spatiotemporal dynamics of intratumoral immune cells reveal the immune landscape in human cancer. Immunity $2013 ; 39: 782-95$.

43. Meshcheryakova A, Tamandl D, Bajna $\varepsilon$, et al. B cells and ectopic follicular structures: novel players in anti-tumor programming with prognostic power for patients with metastatic colorectal cancer. PLoS One 2014 ; 9 : e99008.

44. Wirsing AM, Rikardsen $O G$, Steigen $S E$, et al. Characterisation and prognostic value of tertiary lymphoid structures in oral squamous cell carcinoma. BMC Clin Pathol $2014 ; 14$ : 38.

45. Shalapour $\mathrm{S}$, Lin XJ, Bastian IN, et al. Inflammation-induced IgA $\mathrm{A}^{+}$cells dismantle anti-liver cancer immunity. Nature 2017 ; 551 : 340-5.

\section{TIRÉS À PART}

H. Kaplon et M.-C. Dieu-Nosjean 